\title{
34. Artificial Induction of Pseudencephaly, Short-tail, Taillessness, Myelencephalic Blebs and Some Fissure Formations (Phenocopies) of Mice*)
}

\author{
By Ujihiro MURAKAMI**) \\ Murakami Laboratory of the Research Institute of \\ Environmental Medicine, Nagoya University \\ (Comm. by S. Katsunuma, M.J.A., March 12, 1953)
}

1. Introduction: It has been noticed that certain congenital malformations of mammals and man, which have been thought to be hereditary, have been shown to be caused either clinically or experimentally by a number of abnormal intrauterine environments such as irradiation of X-rays, infections and chronic malnutrition of pregnant mothers.

Certain X-ray mutations and mechanisms of manifestation of some congenital malformations of rodents have been studied by Bagg ('29) ${ }^{1 \text { ), }}$ Bonnevie('34) ${ }^{2}$, Snell et al.('34) ${ }^{3)}$, and phenocopies of rodents caused by X-ray irradiations studied by Kosaka ('28), Job et al. ('35), Kaven ('38), Warkany and Schraffenberger('47), Russell('50) ${ }^{4)}$, et al., but no experiments were made except by Katsunuma('48) ${ }^{5)}$ who placed its significance upon the placenta which forms a distinguishing trait of developmental environment in higher mammals.

However, some abnormalities were seen by Gillaman, Gilbert, Gillman and Spence $\left({ }^{\prime} 48\right)^{6)}$ according to the process like Katsunuma, but their experiment concerned another aspect and they placed no attention upon the rôle of the placenta.

Katsunuma noted that when he administrated vital stains to pregnant rabbits, guinea pigs and rats, in the form of lithioncarmin, trypan red or trypan blue etc., he noticed certain macroscopic abnormalities, such as pseudencephaly, spina bifida anophthalmia, harelip, heart abnormalities etc. in their offspring following full term or premature births, or at autopsy of the mother animals, and he laid stress on placental function which becomes abnormal due to these vital staining dyes.

Gillman et al. mated male rats with bleeding females and injected the latter with trypan blue at 14 day intervals and produced the

*) The outline of this report was presented at the 23rd (1951) and the 24th (1952) meetings of the Genetic Society of Japan.

**) The author wishes to express his deep debt to Dr. Seizo Katsunuma, the President of Nagoya University, for his direction in this study. 
following abnormalities: Hydrocephalus, spina bifida, tail defect, eye defect, ear defect, meningocele, cranioschisis, harelip and cleft palate etc.

Hamburgh ('52) injected trypan blue solution subcutaneously into pregant mice seven to eight days following fertilization and saw two series of abnormalities, namely abnormalities of the anterior and posterior axial regions.

Waddington and Carter ('52) ${ }^{8)}$ stated that when they treated mice 7 days following fertilization with subcutaneous injection of $\frac{1}{2}$ cc. of $1 \%$ trypan blue solution, after 24 hours, embryos were retarded and subectodermal belbs near the somites were produced and after 48 hours, the pericardium became abnormally large and axial turning processes were disturbed, and $10 \frac{1}{2}$ days of gestation the medullary tubes in many cases were not closed and the animals usually die, $12_{2}^{\mathbf{1}}$ days of gestation etc.

The author continued the experiment of Katsunuma and determined the critical period at which the previously mentioned abnormalities are produced, also the mechanism of production of serious changes in the early embryos due to abnormal intrauterine environment. One preliminary report was made by this author in 1951 ,**) ${ }^{\text {9) }}$ using lithioncarmin.

The following report will include the result of the abnormal effect upon embryos in early embryonic stages caused by intravenous injection of trypan blue solution.

2. Methods and Results: Methods and results are in Table I.

3. Comments and Resume: The author injected intravenously, lithioncarmin or trypan blue into early pregnant mice ; and there resulted many abnormal embryos which belong to some phenocopies of several hereditary abnormalities. They were: Pseudencephaly (Fig. 1), distention of the central nervous system (Fig. 2), tortuosities (Fig. 3), and malclosures of the medullary tube (Fig. 4), flexion of the medullary tube, short-tail or taillessness (Fig. 5), flexed tail, blebs bearing resemblance to the myelencephalic blebs (Fig. 6), median harelip associated with pseudencephaly (Fig. 7), ectopia cordis associated with a cranial bleb (Fig. 8), myelocystomenigocele (Fig. 9), severe medullary tube abnormalities associated with an abnormally large heart region (pericardium) etc.

These abnormalities manifested themselves regardless of the qualitative differences in the vital staining dyes used. Most of them are abnormalities of the nervous system and fissure formation. It is assumed that some excessive developmental processes may have been produced.

Under the aforesaid abnormalities there are found various condi- 
tions belonging to the undermentioned ranks crossing each other: Partial flexion of the medullary tube-malclosure or flexion of the medullary tube-tortuosity of the medullary

tube $<$ pseudencephaly.
distention of the central nervous system.

In the cases of "distention" the coverings of the brains are usually formed small and narrow.

The formation of the face region is also retarded, and these embryos usually die during the 11-12th embryonic days (Fig. 10), and in some of them the notochord seemed to disappear (Fig. 11).

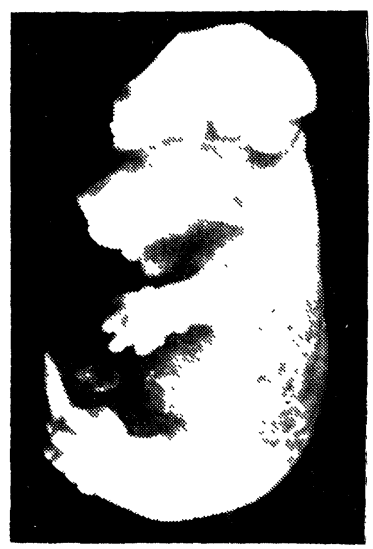

Fig. 1

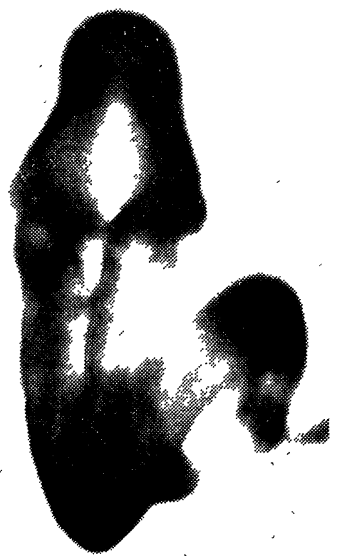

Fig. 4

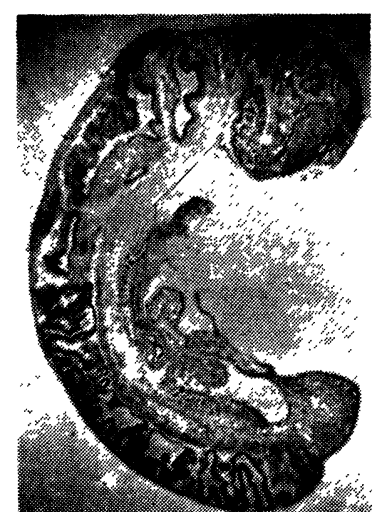

Fig. 2

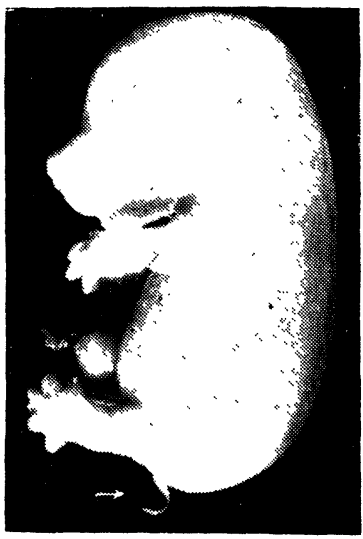

Fig. 5

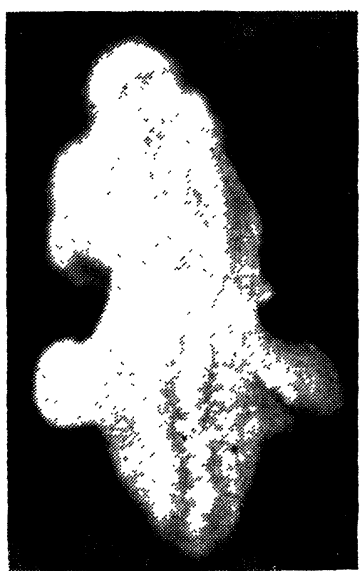

Fig. 3

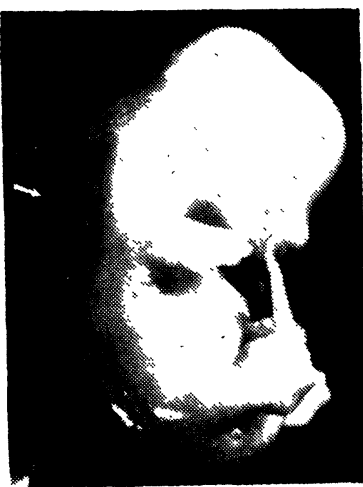

Fig 6.

Fig. 1. No. 87-1: 16th embryonic day; pseudencephaly in highest degree

Fig. 2. No. T304-2: 12th embryonic day; sagittal section of a "distention". Many folds are found in the brain. The medullary tube became tortuous

Fig. 3. No. 136: 13th embryonic day; tortuosity of the medullary tube

Fig. 4. No. T322-1: 11th embryonic day; the closure of the medullary tube was retarded, malclosed lips were somewhat tortuous. Permeable preparation

Fig. 5. No. T363 $3_{-5}$ : 16th embryonic day; the retarded tail looks like a pig tail (taillesseness) $(\rightarrow)$

Fig. 6. No. $85_{-6}$ : 13th embryonic day; back blister, which is pointed out by arrow

It has been reported by Snell et al. in pseudencephaly, and in short- 
tail or taillessness by Chesley ${ }^{10}$, that distentions of the central nervous system are often observed. From the fact that these abnormal conditions were all obtained in the author's experiments, it may be supposed that there exist a close relationship, embryologically-pathologically between them.

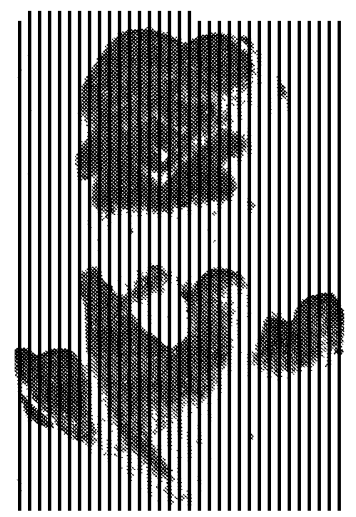

F'ig. 7

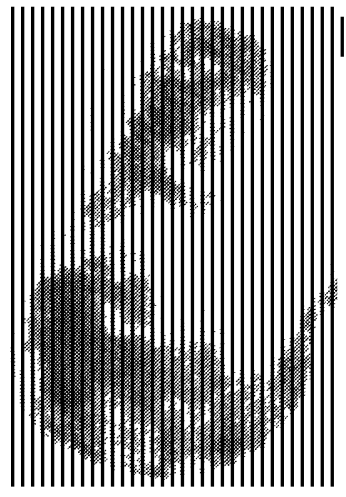

Fig. 10

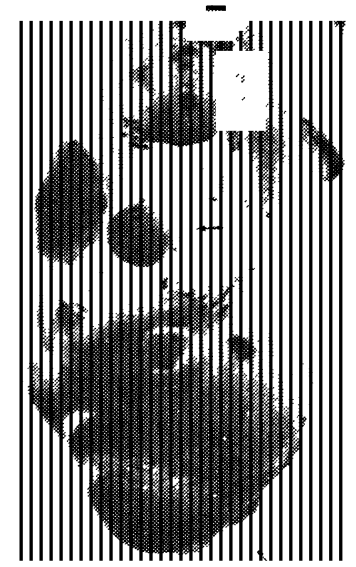

F'ig. 8

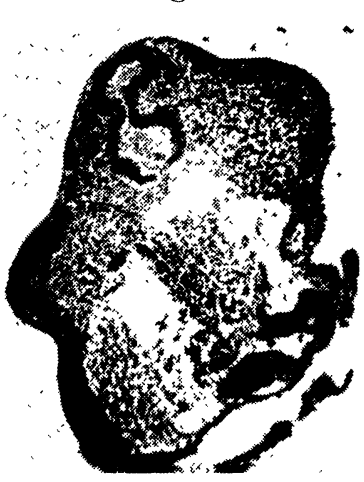

Fig. 11

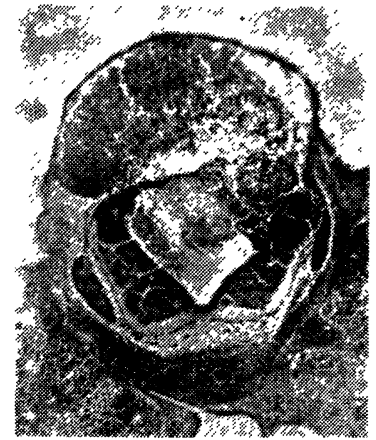

Fig. 9

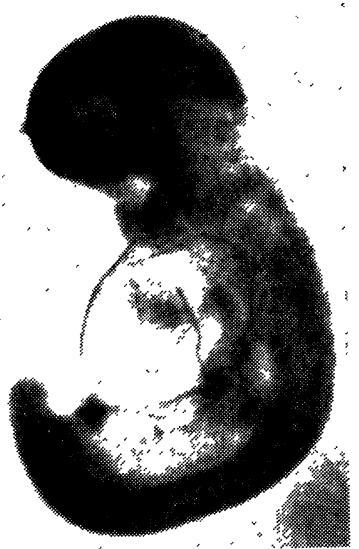

Fig. 12

Fig. 7. No. T336-1 :

13th embryonic day; pseud-

encephaly associated with median harelip

Fig. 8. No. T337-1: 13th embryonic day; ectopia cordis $(\rightarrow)$ associated with cranial blister (\)

Fig. 9. Normal section to the medullary tube in the embryo No. $153_{-1}$ (13th embryonic day). The sac of spinal meninges is filled with blod, the medullary tube is cleft

Fig. 10. No. $85_{-7}$ : 12th embryonic day; "distention", the head region, especially the formation of forehead, maxilla and mandible were retarded

Fig. 11. Cross section of a "distention" (No. T333-2, 10th embryonic day: Notochord not seen

Fig. 12. No. T379-6 : 11th embryonic day ; "distention" associated with large pericardium. Permeable preparation.

On the other hand, the fact that there were also obtained embryos with blisters, like the "myelencephalic blebs", by the same treatment suggests the existence of some common process in the manifestation of hereditary pseudencephaly, short-tail or taillessness 
and myelencephalic blebs, which originate from different genes. They offer some significant problems in embryological genetics, on the mechanism of gene manifestations.

The above-mentioned important abnormalities were most frequently observed in the group treated with intravenous injection of 0.1 cc. of $1 \%$ trypan blue solution on the 8 th day, probably corresponding to days as numbered in this experiment more exactly about $8 \frac{1}{2}$ day of gestation ( 8 of 26 pregnant mice had pseudencephlic embryos, 1 median harelip and 1 extopia cordis) which fall into presomite or early somite stadium. In the group trated on the 7 th day (about $7 \frac{\mathbf{1}}{\mathbf{2}}$ day) of gestation besides the above-mentioned abnormalities, many severe medullary tube abnormalities associated with large pericardium were seen(Fig.12).

Therefore, the critical period of formation of these serious abnormalities, especially of pseudencephaly and other fissure formations, should fall on the $8 \frac{1}{2}$ day of gestation, and that of pseudencephaly is precisely that stage in development to which Bonnevie ${ }^{11)}$ has traced the inherited anomaly. It is also similar to the results caused by $\mathrm{X}$ ray irradiations as Grüneberg ${ }^{12)}$ has noticed.

When we administrated vital staining dyes to mice on the 7 th to 9th pregnant days, the trophoderm, the wall of the blastcyst, were vitally stained and looked pink (by lithioncarmin) or blue (by trypan blue) but these vitally staining dyes are strictly restricted to the yolk sac membrane and the embryo proper is never vitally stained. The functions of the placenta anlage where some conversion might be involved by vitalstaining, should be studied. This problem will be discussed in further reports.

\section{References}

1) Bagg, H. J.: Am. J. Anat., 43, 167-219 (1929).

2) Bonnevie, K.: J. Exp. Zool., 67, 443-520 (1934). (1934).

3) Snell, G. D., Bodemann, E., and Hollander, W.: J. Exp. Zool., 67, 93-104

4) Russell, L. B.: J. Exp. Zool., 114, 545-602 (1950).

5) Katsunuma, S. : Proc. Japan Acad., 24, 47-49 (1948).

6) Gillman, J., Gilbert, Ch., Gillman, Th., and Spence, I.: S. Afr. J. Med. Sci., 13, 47-90 (1948).

7) Hamburgh, M. : Nature, 169, 27 (1952).

8) Waddington, C. H. and Carter, T. C. : Nature, 169, 27-28 (1952).

9) Murakami, U.: Jap. Jour. Genet., 27, 176-184 (1952).

10) Chesley, P.: J. Exp. Zool., 70, 429-456 (1935).

11) Bonnevie, K.: Handb. Erbbiol. Mensch., Bd. I. Berl. (1940).

12) Grüneberg, H.: The Genetics of the Mouse. 2nd Ed. Hague (1952). 